\title{
Effect of red blood cell storage time on markers of hemolysis and inflammation in transfused very low birth weight infants
}

\author{
Tamara G. Kalhan ${ }^{1}$, David A. Bateman ${ }^{2}$, Rakhee M. Bowker ${ }^{3}$, Eldad A. Hod ${ }^{4}$ and Sudha Kashyap ${ }^{2}$
}

BACKGROUND: Prolonged storage of transfused red blood cells (RBCs) is associated with hemolysis in healthy adults and inflammation in animal models. We aimed to determine whether storage duration affects markers of hemolysis (e.g., serum bilirubin, iron, and non-transferrin-bound iron (NTBI)) and inflammation (e.g., interleukin (IL)-8 and monocyte chemoattractant protein (MCP)-1) in transfused very low birth weight (VLBW) infants.

METHODS: Blood samples from 23 independent transfusion events were collected by heel stick before and $2-6 \mathrm{~h}$ after transfusion.

RESULTS: Serum iron, total bilirubin, NTBI, and MCP-1 levels were significantly increased after transfusion of RBCs $(P<0.05$ for each comparison). The storage age of transfused RBCs positively correlated with increases in NTBI following transfusion $\left(P<0.001 ; R^{2}=0.44\right)$. No associations between storage duration and changes in the other analytes were observed.

CONCLUSION: Transfusion of RBCS into VLBW infants is associated with increased markers of hemolysis and the inflammatory chemokine MCP-1. RBC-storage duration only correlated with increases in NTBI levels following transfusion. NTBI was only observed in healthy adults following 35 days of storage; however, this study suggests that VLBW infants are potentially more susceptible to produce this pathological form of iron, with increased levels observed after transfusion of only 20-day-old RBCs.

$T$ ransfusions of packed red blood cells (RBCs) are lifesaving and vital for providing care to very low birth weight (VLBW; $<1,250 \mathrm{~g}$ ) neonates. These infants require transfusions throughout their hospital stay primarily because of anemia of prematurity, which results from diminished RBC production, shortened RBC lifespan, and iatrogenic blood loss from frequent lab draws required for monitoring (1). At our center, and many others, infants are regularly exposed to older blood products because of the current policy of using dedicated blood donors. This policy designates a specific packed $\mathrm{RBC}$ unit, derived from one donor, to one infant, from which the infant receives RBCs each time a transfusion is required until the expiration date. This policy was initially instituted to reduce exposure to multiple donors and decrease transmission of viral pathogens (2). A consequence of this practice is that neonates receive older, stored RBCs with each subsequent transfusion. Several retrospective studies suggested that certain morbidities, such as necrotizing enterocolitis, sepsis, bronchopulmonary dysplasia, and retinopathy of prematurity, may be associated with $\mathrm{RBC}$ transfusions (3-5); in contrast, other studies did not observe such associations $(6,7)$.

In the United States, the Food and Drug Administration mandates that RBCs may be stored for a maximum of 42 days (8). Approximately half of transfused RBC units are stored for 21 days or longer $(9,10)$. During storage, RBCs undergo mechanical and biochemical changes that reduce their recovery in vivo; this is termed the "RBC-storage lesion" $(11,12)$. Damaged RBCs are rapidly cleared from the circulation by reticuloendothelial macrophages, mostly within the first hour post transfusion (13). The catabolized hemoglobin iron is then released and it binds to circulating transferrin in plasma; however, when the rate of iron egress from the macrophage exceeds transferrin-binding capacity, non-transferrin-bound iron (NTBI) is produced $(14,15)$. NTBI may cause oxidative damage and increase infectious risk (16). Studies in healthy adult human volunteers suggest that NTBI is not produced following transfusion unless the RBCs are stored for 35-42 days (17). Nonetheless, VLBW infants may have impaired hepcidin responses and decreased transferrin levels, resulting in a decreased capacity to handle the iron released following transfusion of older, stored RBCs (18-20). Thus, it is possible that NTBI is observed in transfused neonates following transfusion of RBCs stored for less than 35 days (18).

Furthermore, in murine and canine studies, increased NTBI levels and enhanced pro-inflammatory cytokine responses were observed in animals transfused with older RBCs (21-23). This inflammatory response may contribute to the mechanisms responsible for some adverse effects of RBC transfusions (23). Because the risk of acquiring serious neonatal morbidities may be associated with RBC transfusions, and because

\footnotetext{
${ }^{1}$ Division of Neonatology, Department of Pediatrics, Albert Einstein College of Medicine, Bronx, New York; ${ }^{2}$ Division of Neonatology, Department of Pediatrics, College of Physicians and Surgeons, Columbia University, New York, New York; ${ }^{3}$ Division of Neonatology, Department of Pediatrics, Rush University Medical Center, Chicago, Illinois; ${ }^{4}$ Department of Pathology and Cell Biology, Columbia University Medical Center, New York, New York. Correspondence: Eldad A. Hod (eh2217@cumc.columbia.edu) Received 28 March 2017; accepted 18 June 2017; advance online publication 16 August 2017. doi:10.1038/pr.2017.177
} 
adult and animal studies suggest that longer storage duration before transfusion may increase adverse effects, we examined whether there is a relationship in transfused VLBW infants between the age of stored RBCs and circulating posttransfusion NTBI, serum iron, total bilirubin, and proinflammatory cytokine levels.

\section{METHODS}

\section{Study Participants}

Parents of preterm infants with birth weight less than 1,250 g were approached for consent from August 2013 through December 2014 at the Morgan Stanley Children's Hospital of New York Presbyterian Hospital-Columbia University Medical Center, a level IV Neonatal Intensive Care Unit. Patients were included if inborn or transferred from an outside hospital at less than 7 days of life. Patients were excluded if they had been transfused with platelets or fresh frozen plasma within $4 \mathrm{~h}$ of the tested RBC transfusion or were in a moribund condition. Parental consent was obtained from all study patients upon enrollment.

\section{Study Design}

This single-center, prospective, observational study evaluated the relationship between the age of transfused RBCs and serum iron, total bilirubin, NTBI, and circulating inflammatory cytokines. Standard leukoreduced, irradiated, CMV-negative, packed RBCs stored in an additive saline solution were transfused via a peripheral intravenous line over $4 \mathrm{~h}$, as per the protocol at our center. Twentytwo of twenty-three independent transfusions used RBCs stored in AS-3 (Nutricel) storage solution; one transfusion was of RBCs stored in citrate-phosphate-dextrose-adenine (CPDA-1). All RBC units were standard packs of RBCs obtained from the local supplier; in most cases, the parent bag was irradiated before aliquoting into syringes for administration into the neonates in the study as needed. Transfusions were given at the discretion of the neonatology medical team, among whom transfusion practices are similar. The general transfusion threshold practice for VLBW infants in the NICU is to maintain hematocrit $>35 \%$ for those with severe cardiopulmonary diseases requiring mechanical ventilation with significant oxygen requirement in the first week of life, $>30 \%$ for moderate cardiopulmonary disease and for major surgery in hemodynamically stable infants, and $>20 \%$ for growing stable infants without any oxygen requirement (the thresholds maybe slightly higher for extremely low birth weight ( $<1,000 \mathrm{~g})$ infants). A pre-transfusion,

Table 1. Patient demographics

\begin{tabular}{lc}
\hline Characteristic & Value $(N=23)$ \\
\hline Race—no. (\%) & $6(26 \%)$ \\
Caucasian & $5(22 \%)$ \\
African-American & $10(44 \%)$ \\
Hispanic & $1(4 \%)$ \\
Asian & $1(4 \%)$ \\
Mixed race & \\
& $8(35 \%)$ \\
Male, $n$ (\%) & $745(474-1020)$ \\
Birth weight (g)—median (range) & $26(23-30)$ \\
Gestational age at birth (weeks)—median (range) & $11(4-45)$ \\
Age of infant at transfusion (days)—median (range) & $9(1-34)$ \\
Age of transfused RBCs (days)—median (range) & \\
\hline RBC, red blood cell. &
\end{tabular}

$1 \mathrm{ml}$ heel stick or venous blood sample was drawn within $1 \mathrm{~h}$ before transfusion and another $1 \mathrm{ml}$ sample was drawn at 2-6 h (target $4 \mathrm{~h}$ ) post transfusion. Infants were transfused between 10 and $20 \mathrm{ml} / \mathrm{kg}$ of blood as clinically indicated $(17.0 \pm 3.3 \mathrm{ml} / \mathrm{kg} ;$ mean $\pm \mathrm{SD})$ and an additional $2 \mathrm{ml}$ to account for the blood obtained for this study. The study protocol was approved by the Columbia University Medical Center Institutional Review Board.

\section{Laboratory Methods}

Samples were collected in an additive-free, serum separator tube, allowed to clot for $20 \mathrm{~min}$, and then centrifuged at $2,800 \mathrm{~g}$ for $10 \mathrm{~min}$. Serum aliquots were frozen at $-80^{\circ} \mathrm{C}$ until being tested. NTBI was measured using an ultrafiltration colorometric assay $(24,25)$. Serum iron was measured using a colorometric method following the manufacturer's instructions (Iron/TIBC Reagent Set, BQ Kits, San Diego, CA, USA). Bilirubin was measured using the standard clinical laboratory methodology in the New York Presbyterian Hospital Clinical Laboratory. Interleukin (IL)-8 and monocyte chemoattractant protein (MCP)-1 were measured with solid-phase sandwich ELISA (R\&D Systems, Minneapolis, MN, USA) following the manufacturer's instructions.

\section{Data Analysis}

For each study patient, infant and maternal medical records were reviewed and clinical characteristics noted. Power analysis was not performed for this convenience sample. Difference between the means was assessed using paired $t$-test. Correlation with storage age was determined by Pearson linear regression. Multivariable regression was used to test for potential confounding by volume of RBCs transfused $(\mathrm{ml} / \mathrm{kg})$, sepsis/bacteremia (yes/no), and requirement for intubation (yes/no). $P$ value less than 0.05 was considered significant. Prism (GraphPad Software Inc., San Diego, CA) was used for all analyses.

\section{RESULTS}

\section{Demographics and Clinical Characteristics}

From August 2013 through December 2014, we enrolled 23 infants transfused with RBCs. Clinical characteristics of these infants are provided in Table 1. Median gestational age was 26 weeks (range 23-30 weeks) and median birth weight was $745 \mathrm{~g}$. Median pre-transfusion hematocrit was $29 \%$ and median storage age of transfused RBCs was 9 days (range 1-34 days).

\section{Effect of RBC-Storage Age on Markers of Extravascular Hemolysis}

The study subjects demonstrated increased serum total bilirubin (mean change $=0.45 \mathrm{mg} / \mathrm{dl} ; P<0.05$ ), iron (mean change $=21.4 \mu \mathrm{g} / \mathrm{dl} ; P<0.01$ ), and NTBI (mean change $=$ $0.37 \mu \mathrm{M} ; P<0.05)$ levels post transfusion, as compared with the levels pre-transfusion (Figure 1). However, only the change in circulating NTBI level was significantly and positively associated with RBC-storage duration $(P<0.001$, $R^{2}=0.44$; Figure 1f). This relationship was not affected by volume of RBCs transfused, presence of sepsis/bacteremia at the time of transfusion, or requirement for intubation in multivariable regression (data not shown). Furthermore, the lower limit of detection of the NTBI assay used is $0.87 \mu \mathrm{M}$ (ref. 25). All (3/3) transfusions of RBCs stored for $\geq 20$ days and $10 \%(2 / 20)$ of transfusions of RBC stored for $<20$ days were above the lower limit of detection at $4 \mathrm{~h}$ after transfusion and below this limit before transfusion, respectively $(P<0.01$ by Fisher's Exact Test). Of note, the three transfusions of RBC 


\section{Articles $\mid$ Kalhan et al.}

a

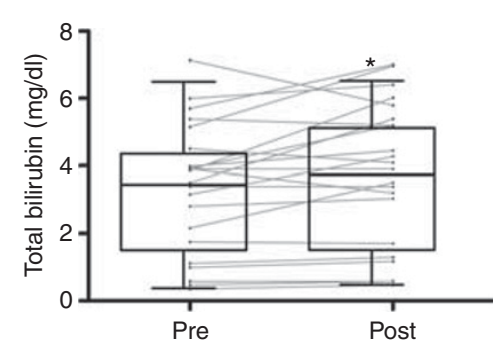

b
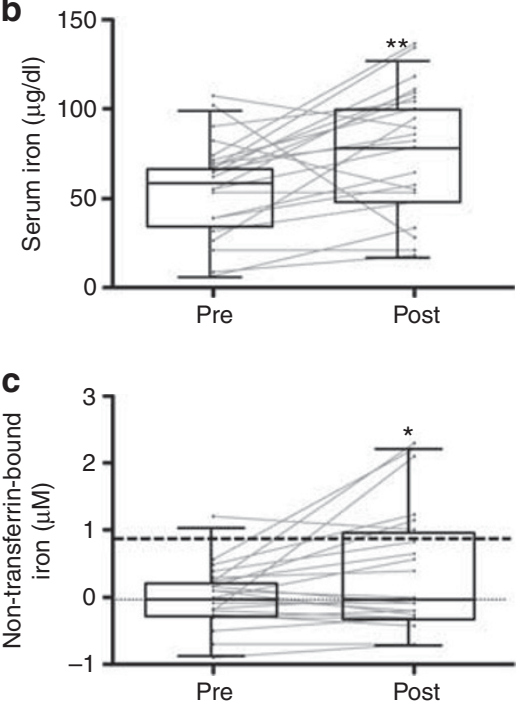

d
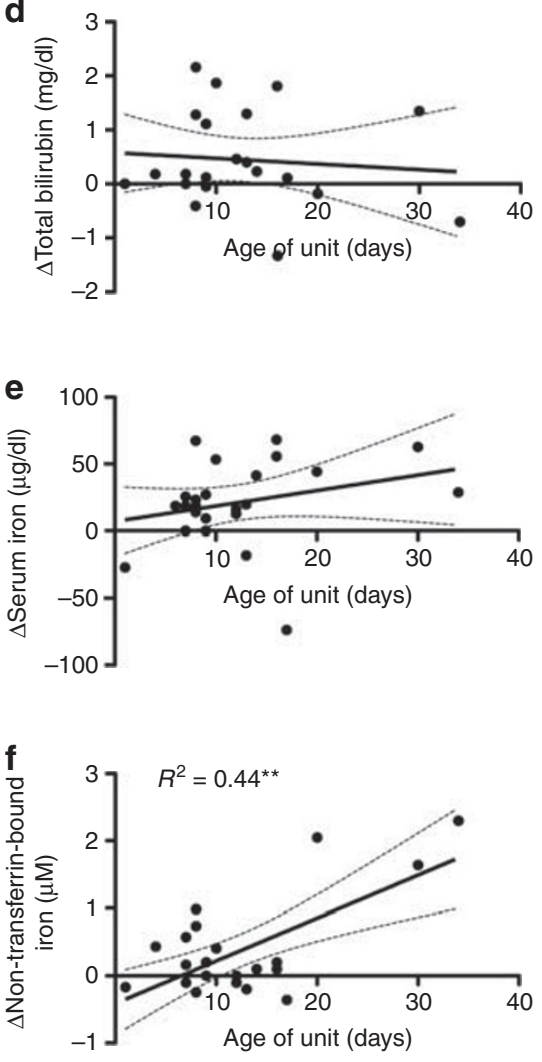

Figure 1. Effects of RBC transfusion on markers of hemolysis and iron status. Pre- and post-transfusion serum total bilirubin (a), iron (b), and non-transferrin-bound iron (c) levels. Standard box and whisker plots in which the box represents the 25th, median, and 75th percentile and the whiskers represent 5th and 95th percentile for the value. Gray lines represent each subject's pre- and post-transfusion analyte level. Linear regression of the age of the transfused RBC unit in days vs. the change in analyte level from pre- to post transfusion for serum total bilirubin (d), iron (e), and non-transferrin-bound iron (f); dark dashed line denotes lower limit of detection for non-transferrin-bound iron $(0.87 \mu \mathrm{M}) .{ }^{*} P<0.05,{ }^{* *} P<0.01$. RBC, red blood cell.

stored for $\geqslant 20$ days also were stored for 13,21 , and 28 days following irradiation.

\section{Effect of RBC-Storage Age on Pro-inflammatory Cytokines}

The VLBW neonates had increased serum MCP-1 levels post transfusion (mean change $=386.5 \mu \mathrm{g} / \mathrm{ml} ; \quad P<0.001$; Figure 2a); however, no significant changes in IL-8 levels were observed (Figure 2b). Furthermore, changes in MCP-1 and IL-8 levels did not correlate with the RBC-storage duration (Figure $2 \mathbf{c}, \mathbf{d}$ ). These relationships were not affected by volume of RBCs transfused, presence of sepsis/bacteremia at the time of transfusion, or requirement for intubation in multivariable regression (data not shown).

\section{DISCUSSION}

This study demonstrates that transfusion of RBCs after longer refrigerator-storage duration increases circulating NTBI in VLBW preterm neonates. A recent study in healthy adults suggests that NTBI is not observed after transfusion until the RBCs are stored for $\geq 35$ days (17). Substantial increases in circulating NTBI were also not observed in critically ill children until the final 2 weeks of storage (26). Taken together, these results suggest that preterm neonates are more susceptible in producing NTBI following RBC transfusion, and this pathologic form of iron can be observed robustly after transfusion of blood stored for only 20 days.

Circulating NTBI is iron that is neither bound to the physiologic iron-transport protein, transferrin, nor is a constituent of heme or ferritin $(14,15)$. In healthy individuals, little, if any, circulating NTBI is ever observed. Iron enters the plasma principally by recycling hemoglobin-iron derived from senescent erythrocytes by reticuloendothelial macrophages in the spleen, liver, and bone marrow (27). During routine refrigerated storage, a proportion of the RBCs become irreversibly damaged and are cleared by these reticuloendothelial macrophages (28). Using Food and Drug Administration criteria, on average, up to $25 \%$ of the transfused RBCs may be rapidly cleared; because most of these damaged RBCs are cleared within the first hour of transfusion (13), the rate of iron influx into plasma can exceed the rate of iron acquisition by transferrin. This results in the production of circulating NTBI, which is a heterogeneous assortment of iron 

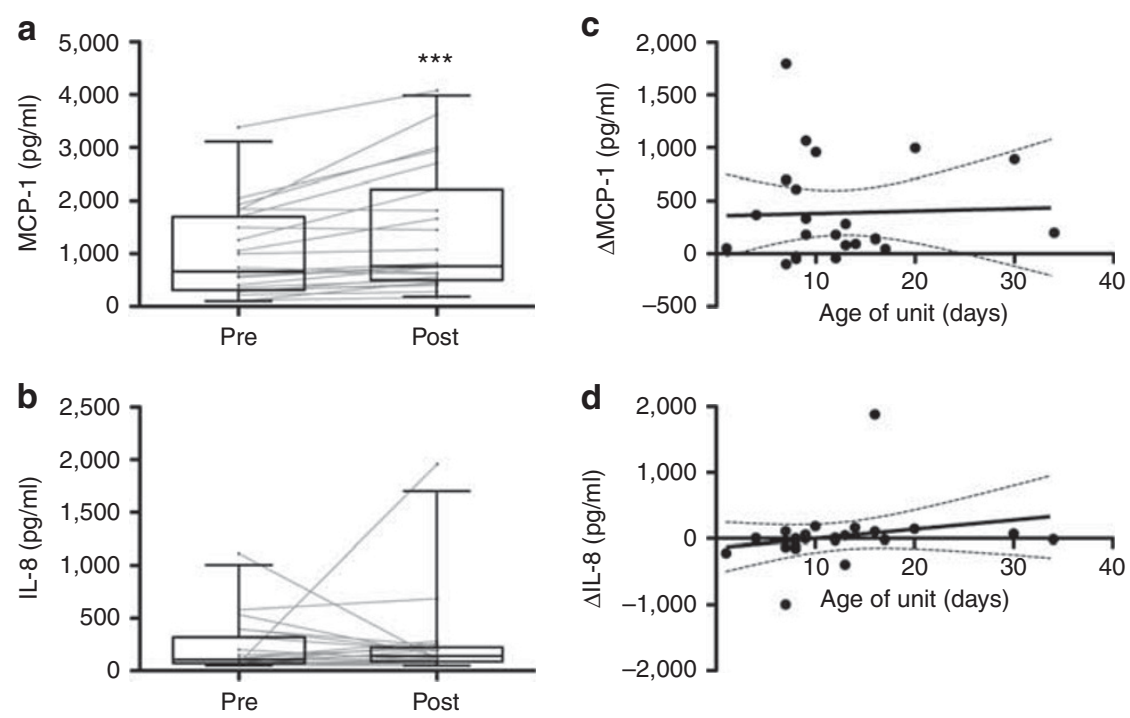

Figure 2. Effects of RBC transfusion on pro-inflammatory chemo/cytokine levels. Pre- and post-transfusion circulating levels of MCP-1 (a) and IL-8 (b). ${ }^{* * *} P<0.001$. Linear regression of the age of the transfused RBC unit in days vs. the change in analyte level from pre- to post transfusion for MCP-1 (c) and IL-8 (d). IL, interleukin; MCP, monocyte chemoattractant; RBC, red blood cell.

complexes (e.g., with albumin or citrate) (14). Neonates may be particularly prone to produce NTBI due to decreased concentrations of circulating transferrin and impaired hepcidin responses (18-20).

Accumulating evidence indicates that NTBI can cause adverse effects (16). In animal studies, even short durations of circulating NTBI enhanced the virulence of specific bloodstream pathogens $(22,23,29,30)$. In addition, increased NTBI in human serum samples obtained after transfusing 6-weekold RBCs enhanced proliferation of a pathogenic strain of Escherichia coli in vitro (24). Similarly, following oral iron supplementation, the increased serum iron enhanced proliferation in vitro of several bacterial pathogens, including E. coli (31). Circulating NTBI is also associated with increased risks of infectious complications following myelosuppression for acute leukemia, where patients with plasma NTBI levels $>2 \mu \mathrm{M}$, like those observed in this study after transfusing RBCs stored for $>20$ days, had a higher risk of Gramnegative sepsis $(P=0.0004$; (ref. 32)). This raises the possibility that the NTBI produced following some RBC transfusions may be responsible for the purported association between RBC transfusion and necrotizing enterocolitis $(3,4)$. Indeed, recent studies identified uropathogenic E. coli, a highly ferrophilic organism, as a risk factor for necrotizing enterocolitis (33). Finally, by participating in Fenton chemistry, NTBI causes oxidative damage, cytotoxicity, and enhancement of endothelial expression of adhesion molecules $(14,15,34,35)$. Indeed, Stark et al. (36) showed that malondialdehyde, a marker of oxidative stress, correlates with NTBI levels observed following RBC transfusions in VLBW infants.

Despite the potential for NTBI to cause harm, the randomized clinical trials assessing the benefit of a fresher
RBC transfusion policy have not observed clinically significant differences in outcome. In a similar population as the VLBW infants in this study, the Age of Red Blood in Premature Infants Trial was a double-blind, randomized controlled trial in 377 premature infants with birth weights $<1,250$ g randomized to a transfusion policy of "fresh" RBCs, stored for 7 days or less, as compared with the standard of care (7). The overall conclusion was that a fresh RBC transfusion policy did not improve outcomes in premature, VLBW infants. Notably, the median age of the standard of care RBCs in that trial was 14.6 days. In contrast, our study, in combination with others $(36,37)$, suggests that there is very little evidence of NTBI production after transfusing RBCs stored for less than 20 days. Thus, to the extent that NTBI is responsible for adverse effects of transfusing older, stored RBCs, an observable clinical difference would not be expected between the groups in the Age of Red Blood in Premature Infants Trial. Furthermore, irradiation damages RBCs and prolonged storage following irradiation exacerbates the RBCstorage lesion $(38,39)$. Thus, the practice of irradiating the parent bag before storage as opposed to the aliquoted syringe before transfusion may be responsible for exacerbating the hemolysis observed following RBCs stored for longer than 20 days. It was not possible to disentangle whether the prolonged storage following irradiation is responsible for the increases in NTBI observed in this observational study. Therefore, additional studies are recommended to assess the safety of transfusing RBCs stored for longer than 20 days along with the effects of storage following irradiation in this vulnerable patient population.

Following transfusion of longer stored RBCs, a pro-inflammatory cytokine response was observed in animal models (21), (23). Furthermore, these studies(23) suggest that 


\section{Articles | Kalhan et al.}

intact, but damaged, RBCs are responsible for this inflammatory response. Similar to other studies (37), posttransfusion MCP-1 levels were increased in our study; however, a correlation with storage age was not examined previously. Given that increases in MCP-1 levels in our study did not correlate with increased RBC-storage duration, these increases seem unrelated to older RBC transfusion and may be caused by a confounding factor. For example, the first heel stick pre-transfusion may cause localized inflammation that is then measured by the second post-transfusion heel stick. Without an appropriate control group of non-transfused VLBW infants (e.g., those getting saline or albumin infusions instead of transfusion), this issue is difficult to resolve. Unfortunately, for ethical reasons, it is very difficult to identify and justify such a control group.

Other limitations of our study include the limited laboratory outcomes assessed because of the limited amount of serum collected from VLBW infants. Furthermore, this single-center study had a convenience sample of only 23 neonates. The challenges involved in obtaining informed consent and collecting these precious samples make it difficult to perform larger, more adequately powered studies. However, our main NTBI outcome is confirmed by other small studies in VLBW infants $(36,40)$. Finally, some infants were transfused before obtaining consent for this study and only samples from transfusions following informed consent were collected; thus, not all samples are from the first transfusion event of life.

In conclusion, this study confirms that transfusions of RBCs after longer storage durations are associated with increased NTBI, particularly after 20 days of storage. Although the clinical consequences of these findings remain to be determined, it is evident that the responses to $\mathrm{RBC}$ transfusion in VLBW preterm infants differ from those observed in healthy adult volunteers (17) and in older, critically ill pediatric patients (26). Given the lack of clinical evidence supporting the safety in neonates of RBC transfusions stored for longer than 20 days (7), the policy of using the same dedicated donor unit per neonate, in which subsequent aliquots are stored for longer durations, should be revisited, paying particular attention to those units stored for longer than 20 days.

\section{AUTHOR CONTRIBUTIONS}

T.G.K., R.A.K., E.A.H., and S.K. designed the study. T.G.K. and S.K. acquired the data. T.G.K. and E.A.H. performed laboratory testing of samples. T.G.K., E.A.H., S.K., and D.A.B. interpreted the data and performed statistical analysis. T.G.K. drafted the manuscript; all authors edited drafts and reviewed the final version of the manuscript.

\section{STATEMENT OF FINANCIAL SUPPORT}

This publication was supported by the National Center for Advancing Translational Sciences, National Institutes of Health, through Grant Number UL1TR000040.

\section{DISCLAIMER}

The content is solely the responsibility of the authors and does not necessarily represent the official views of the $\mathrm{NIH}$.
Disclosure: The authors declare no conflict of interest.

\section{REFERENCES}

1. Strauss RG. Anaemia of prematurity: pathophysiology and treatment. Blood Rev 2010; 24:221-5.

2. Mangel J, Goldman M, Garcia C, Spurll G. Reduction of donor exposures in premature infants by the use of designated adenine-saline preserved split red blood cell packs. J Perinatol 2001;21:363-7.

3. Mally P, Golombek SG, Mishra R, et al. Association of necrotizing enterocolitis with elective packed red blood cell transfusions in stable, growing, premature neonates. Am J Perinatol 2006;23:451-8.

4. Paul DA, Mackley A, Novitsky A, Zhao Y, Brooks A, Locke RG. Increased odds of necrotizing enterocolitis after transfusion of red blood cells in premature infants. Pediatrics 2011;127:635-41.

5. Zhang Z, Huang X, Lu H. Association between red blood cell transfusion and bronchopulmonary dysplasia in preterm infants. Sci Rep 2014;4:4340.

6. Valieva OA, Strandjord TP, Mayock DE, Juul SE. Effects of transfusions in extremely low birth weight infants: a retrospective study. J Pediatr 2009;155:331-7 e331.

7. Fergusson DA, Hebert P, Hogan DL, et al. Effect of fresh red blood cell transfusions on clinical outcomes in premature, very low-birth-weight infants: the ARIPI randomized trial. JAMA 2012;308:1443-51.

8. Dumont LJ, AuBuchon JP. Evaluation of proposed FDA criteria for the evaluation of radiolabeled red cell recovery trials. Transfusion 2008;48: 1053-60.

9. Fontaine MJ, Chung YT, Erhun F, Goodnough LT. Age of blood as a limitation for transfusion: potential impact on blood inventory and availability. Transfusion 2010;50:2233-9.

10. Whitaker B, Rajbhandary S, Kleinman S, Harris A, Kamani N. Trends in United States blood collection and transfusion: results from the 2013 AABB Blood Collection, Utilization, and Patient Blood Management Survey. Transfusion 2016;56:2173-83.

11. Zimring JC. Established and theoretical factors to consider in assessing the red cell storage lesion. Blood 2015;125:2185-90.

12. Glynn SA, Klein HG, Ness PM. The red blood cell storage lesion: the end of the beginning. Transfusion 2016;56:1462-8.

13. Luten M, Roerdinkholder-Stoelwinder B, Schaap NP, de Grip WJ, Bos HJ, Bosman GJ. Survival of red blood cells after transfusion: a comparison between red cells concentrates of different storage periods. Transfusion 2008;48:1478-85.

14. Hider RC. Nature of nontransferrin-bound iron. Eur J Clin Invest 2002;32 (Suppl 1): 50-4.

15. Brissot P, Ropert M, Le Lan C, Loreal O. Non-transferrin bound iron: a key role in iron overload and iron toxicity. Biochim Biophys Acta 2012;1820:403-10.

16. Hod EA, Spitalnik SL. Stored red blood cell transfusions: Iron, inflammation, immunity, and infection. Transfusion 2012;19:84-9.

17. Rapido F, Brittenham GM, Bandyopadhyay S, et al. Prolonged red cell storage before transfusion increases extravascular hemolysis. J Clin Invest 2016;127:375-82.

18. Hirano K, Morinobu T, Kim H, et al. Blood transfusion increases radical promoting non-transferrin bound iron in preterm infants. Arch Dis Child Fetal Neonatal Ed 2001;84:F188-93.

19. Kitajima J, Ohga S, Kinjo T, et al. Serum prohepcidin concentrations at birth and 1 month after birth in premature infants. Pediatr Blood Cancer 2011;56:267-72.

20. Lorenz L, Peter A, Poets CF, Franz AR. A review of cord blood concentrations of iron status parameters to define reference ranges for preterm infants. Neonatology 2013;104:194-202.

21. Callan MB, Patel RT, Rux AH, et al. Transfusion of 28-day-old leucoreduced or non-leucoreduced stored red blood cells induces an inflammatory response in healthy dogs. Vox Sang 2013;105:319-27.

22. Cortes-Puch I, Remy KE, Solomon SB, et al. In a canine pneumonia model of exchange transfusion, altering the age but not the volume of older red blood cells markedly alters outcome. Transfusion 2015;55: 2564-75. 
23. Hod EA, Zhang N, Sokol SA, et al. Transfusion of red blood cells after prolonged storage produces harmful effects that are mediated by iron and inflammation. Blood 2010;115:4284-92.

24. Hod EA, Brittenham GM, Billote GB, et al. Transfusion of human volunteers with older, stored red blood cells produces extravascular hemolysis and circulating non-transferrin-bound iron. Blood 2011;118:6675-82.

25. de Swart L, Hendriks JC, van der Vorm LN, et al. Second international round robin for the quantification of serum non-transferrin-bound iron and labile plasma iron in patients with iron-overload disorders. Haematologica 2016;101:38-45.

26. L'Acqua C, Bandyopadhyay S, Francis RO, et al. Red blood cell transfusion is associated with increased hemolysis and an acute phase response in a subset of critically ill children. Am J Hematol 2015;90:915-20.

27. Andrews NC. Forging a field: the golden age of iron biology. Blood 2008;112:219-30.

28. Wojczyk BS, Kim N, Bandyopadhyay S, et al. Macrophages clear refrigerator storage-damaged RBCs and subsequently secrete cytokines in vivo, but not in vitro, in a murine model. Transfusion 2014;54:3186-97.

29. Solomon SB, Wang D, Sun J, et al. Mortality increases after massive exchange transfusion with older stored blood in canines with experimental pneumonia. Blood 2013;121:1663-72.

30. Prestia K, Bandyopadhyay S, Slate A, et al. Transfusion of stored blood impairs host defenses against Gram-negative pathogens in mice. Transfusion 2014;54:2842-51.

31. Cross JH, Bradbury RS, Fulford AJ, et al. Oral iron acutely elevates bacterial growth in human serum. Sci Rep 2015;5:16670.
32. Belotti A, Duca L, Borin L, et al. Non transferrin bound iron (NTBI) in acute leukemias throughout conventional intensive chemotherapy: kinetics of its appearance and potential predictive role in infectious complications. Leuk Res 2015;39:88-91.

33. Ward DV, Scholz M, Zolfo M, et al. Metagenomic sequencing with strain-level resolution implicates uropathogenic e. coli in necrotizing enterocolitis and mortality in preterm infants. Cell Rep 2016;14:2912-4.

34. Hershko C. Mechanism of iron toxicity. Food Nutr Bull 2007;28:S500-9.

35. Mollet IG, Patel D, Govani FS, et al. Low dose iron treatments induce a DNA damage response in human endothelial cells within minutes. PLoS ONE 2016;11:e0147990.

36. Stark MJ, Keir AK, Andersen CC. Does non-transferrin bound iron contribute to transfusion related immune-modulation in preterms? Arch Dis Child Fetal Neonatal Ed 2013;98:F424-9.

37. Keir AK, McPhee AJ, Andersen CC, Stark MJ. Plasma cytokines and markers of endothelial activation increase after packed red blood cell transfusion in the preterm infant. Pediatr Res 2013;73:75-9.

38. Davey RJ, McCoy NC, Yu M, Sullivan JA, Spiegel DM, Leitman SF. The effect of prestorage irradiation on posttransfusion red cell survival. Transfusion 1992;32:525-8.

39. Moroff G, Holme S, AuBuchon JP, Heaton WA, Sweeney JD, Friedman LI. Viability and in vitro properties of AS-1 red cells after gamma irradiation. Transfusion 1999;39:128-34.

40. Dani C, Martelli E, Bertini G, et al. Effect of blood transfusions on oxidative stress in preterm infants. Arch Dis Child Fetal Neonatal Ed 2004;89:F408-11. 